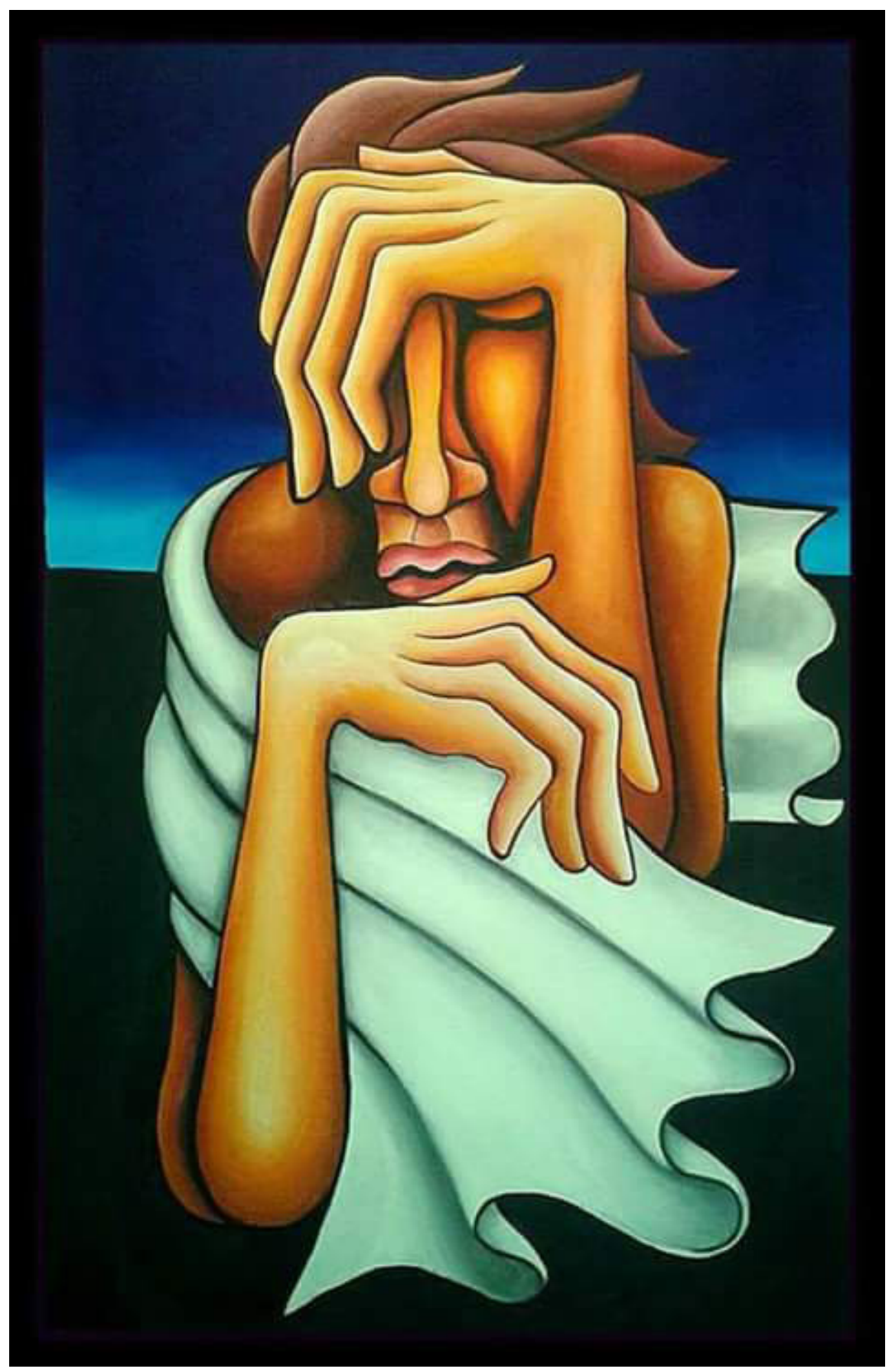

Artista: Heri Tapia 


\title{
Aventura Tecnológica. Experiencia de la Institución Educativa Ricaurte
}

\author{
TECHNOLOGICAL ADVENTURE. AN EXPERIENCE AT THE INSTITUCIÓN \\ EDUCATIVA RICAURTE \\ AVENTURA TECNOLÓGICA. EXPERIÊNCIA DA INSTITUIÇÃO EDUCATIVA \\ RICAUTE
}

\author{
Andrea Milena Gallo Lozano ${ }^{*}$
}

\section{Resumen}

La educación de la actualidad se encuentra en plena transformación, producto de una época hipermoderna, inseparable de los procesos de globalización, y estos a su vez, ligados a las nuevas tecnologías. De ahí que, debamos pensar en la alfabetización digital, en nativos e inmigrantes digitales y en la concordancia que llevan dichos procesos con la formación de seres humanos, con formas de pensar y sentir diferente. Dicha situación es vivida a diario en las instalaciones de la Institución Educativa Ricaurte del municipio de Soacha donde directivos, maestros y estudiantes se han dado a la tarea de dejar atrás las metodologías tradicionalistas para explorar nuevas formas de enseñar y aprender, en el intento de cumplir el reto de la sociedad colombiana.

\section{Abstract}

In the present, education is in plain transformation as a result of a hypermodern era, which is tied to globalization processes and new technologies. Hence, we should think about digital literacy, digital natives and digital immigrants, and the concordance between these processes and the formation of human beings with different ways of thinking and feeling. This situation is lived daily at the Institución Educativa Ricaurte, in the municipality of Soacha, where administrators, teachers, and students have been dedicated to leaving behind traditionalist methodologies in order to explore new ways of teaching and learning, seeking to meet the challenges of the Colombian society.

\section{Resumo}

A educação da atualidade está em plena transformação, produto de uma época hipermoderna, inseparável dos processos de globalização, e esses, vinculados às novas tecnologias. Por isso devemos pensar na alfabetização digital, em nativos e imigrantes digitais e na concordância que requerem esses processos com a formação de seres humanos, com maneiras de pensar e sentir diferentes. Essa situação é vivida a diário nas instalações da Instituição Educativa Ricaute do município de Soacha, onde diretivos, professores e estudantes decidiram deixar as metodologias tradicionalistas para explorar novas formas de ensinar e aprender, tentando responder ao desafio da sociedade colombiana.

Fecha de recepción: 08 de agosto de 2015 / Fecha de aprobación: 10 de septiembre de 2015

\footnotetext{
1 * Magíster en Educación-Comunicación, Universidad Distrital Francisco José de Caldas. Institución
} Educativa Compartir, Soacha.

\section{Palabras clave}

Hipermodernidad, nuevas tecnologías, alfabetización digital, nativos e inmigrantes digitales

\section{Key words}

Hypermodernism, new technologies, digital literacy, digital natives, digital immigrants.

\section{Palavras chave}

Hipermodernidade, novas tecnologias, alfabetização digital, nativos e imigrantes digitais 
Durante los últimos cuarenta años, las sociedades han cambiado vertiginosamente gracias a la aparición y el perfeccionamiento de diferentes medios de comunicación como la telefonía celular, los televisores, los satélites y la internet, entre otros. Dichos avances tecnológicos se han extendido alrededor del mundo haciendo que la adquisición y uso de cada uno de ellos sea indispensable. De este modo, el manejo de la información se ha transformado a nivel mundial, pues esta Ilega instantáneamente a casi todos los rincones permitiendo así, la socialización de los propios avances tecnológicos, las problemáticas sociales, políticas, culturales y económicas de cada país.

La inmediatez y la accesibilidad a la información sin restricción alguna han hecho desaparecer fronteras entre países y clases sociales, creando paulatinamente una nueva cultura, la cultura del consumo, tal y como afirma Lipovetsky (2010):

La cultura que caracteriza la época hipermoderna no es ya el conjunto de normas sociales heredadas del pasado y la tradición (la cultura en sentido antropológico), ni siquiera el mundillo de las artes y las letras (alta cultura); se ha convertido en un sector económico en plena expansión, tan considerable que ha acabado por hablarse, y no sin motivo, de "capitalismo cultural". (p.75)

Cabe aclarar que para Lipovetsky (2006) la hipermodernidad corresponde a la época desde comienzos de los noventa hasta nuestros días, caracterizada por una sociedad basada en el mercado, en la técnica y en el individuo, donde este último es completamente independiente; con lo cual se debilitan los grupos sociales como la familia y sistemas de comportamientos y prácticas como la religión y la cultura. Todo busca ser más y más rápido, ser "hiper". Sin embargo, dicha hipermodernidad fue precedida por la posmodernidad, caracterizada por el individuo que busca la autorrealización, que quiere vivir el aquí y el ahora, sin ideales ni tradiciones, contrariamente a la época de las ideologías, las vanguardias y las revoluciones, a la época en que se creía en el futuro, en la ciencia y en la técnica, la época de comienzos del siglo xx, la época modernista.

La hipermodernidad descrita por Lipovetsky no se aleja de la realidad, pues niños, jóvenes y adultos se han visto inmersos en esa sociedad de mercado donde se produce una alta satisfacción por el consumo de productos, de moda, de tecnología, de cine, de televisión, etc. De esta manera, ese hiperconsumo favorece el desarrollo de la globalización, pues los mercados, sociedades y culturas del mundo se unen a través de la progresiva comunicación entre los distintos países transformándose, a su vez, social, económica y políticamente.

Ahora bien, la globalización permite mayor eficiencia y competencia en el mercado, mejora la cooperación internacional, mejora el aprovechamiento y la explotación de los recursos, así como el desarrollo científicotécnico, entre otros. Sin embargo, las transformaciones que surgen a partir de esta no son del todo buenas, pues como respuesta a la hipermodernidad, han surgido problemáticas que afectan a las sociedades de todo el mundo como la instalación de la virtualidad, el ciberespacio, la digitalización, etc.

La virtualidad, por ejemplo, está presente en todos los ámbitos de una sociedad, como el trabajo, la economía, la educación e incluso las relaciones afectivas. "Hoy no se hace nada, por muy complicado o diminuto que sea, sin un ordenador por alguna parte" (Lipovetsky, 2010, p. 85). El mundo virtual puede ser muy útil pero también puede llevar a la superficialidad y a la falta de análisis y criticidad de las situaciones que se dan cotidianamente. Al respecto, Lipovetsky (2010) afirma que "la vida intelectual ha caído, a su vez, en la lógica de la mercadotecnia, la mediatización y el estrellato ilimitado" (p. 115)

En el ciberespacio, se permite un flujo de información sin límites a tal punto, que se ha pasado de una época de información restringida, limitada o no inmediata a una época de sobreinformación. Esta sobrecarga de información y el poco tiempo para su análisis hace que los individuos limiten sus capacidades, se desorienten, confundan o simplemente no sepan qué hacer con toda la información que les llega a diario. Frente a esto, Lipovetsky (2010) declara:

Lo que nos falta no es información, que nos desborda; lo que no tenemos es un método para orientarnos a una distancia analítica y crítica que le dé sentido. Aquí tenemos una de las grandes apuestas de la cultura-mundo: cómo educar a los individuos y formar espíritus libres en un universo que rebosa información. (p. 89) 
Dada esta compleja y acelerada transformación, se hace necesario buscar la mejor estrategia para preparar a la sociedad en el campo de la comunicación y la información de modo tal, que pueda responder satisfactoriamente a dichos cambios. Es decir, una educación que permita enfrentar las nuevas realidades del ser humano, así como el desarrollo de sus habilidades y sus destrezas físicas, intelectuales e incluso emocionales. Así pues, es de vital importancia que los futuros docentes se preparen desde su formación para enfrentar tales retos.

Para dar respuesta a estos desafíos, es indispensable conocer a fondo las implicaciones de enfrentar en forma adecuada el manejo de la información. Para ello, algunos profesionales de la comunicación se han dado a la tarea de identificar el vocabulario preciso para referirse al camino que posiblemente brinde solución a las diferentes problemáticas de esta época hipermoderna. Uno de ellos es Pérez Tornero, quien en su proyecto Promoting Digital Literacy (2004) explica que el término que mejor describe lo que se pretende en el campo educativo y de la comunicación es "alfabetización digital".

Sin embargo, al escuchar el término "alfabetización digital", normalmente se cree que es el simple aprendizaje de cómo manejar los recursos tecnológicos y aprender el nuevo léxico que se utiliza con dichos recursos, cuando en realidad es un proceso más complicado, pues si analizamos cuidadosamente, nos daremos cuenta de que la tecnología es más que el adecuado manejo de aparatos, es toda una transformación intelectual, emocional, social y cultural del ser humano durante el último siglo.

En Colombia, por ejemplo, muchas instituciones educativas tratan de llevar a cabo una "alfabetización digital" que se reduce al conocimiento de las partes del computador, pues en muchas de ellas ni siquiera se tiene acceso a dicho recurso. En otras, existen los recursos, pero los docentes no están preparados para brindar tal "alfabetización" puesto que tampoco ellos la tienen, así que, con frecuencia, los equipos dispuestos para la llamada "innovación tecnológica" ni siquiera son puestos en marcha por miedo a no saber utilizarlos o miedo a dañarlos.
De este modo, las políticas de desarrollo tecnológico propuestas por el Gobierno Nacional -comenzando por la Ley 115 , artículo 23, donde se presenta al área de Tecnología e informática como área fundamental- no se cumplen, pues en la mayoría de colegios públicos pasó a ser un área que es orientada por docentes que no siempre están preparados para ello, o simplemente es considerada como una asignatura para completar cargas académicas de los docentes. Tenemos también el Plan Nacional de Tecnologías de la Información y Comunicación 2008-2019, cuyo objetivo principal es hacer que todos los colombianos hagan un uso eficiente de las tecnologías, aumentando la competitividad del país, sin embargo, hasta el momento no todos los rincones del país poseen los medios físicos (computadores-conectividad), ni educativos en términos tecnológicos, que les permita alcanzar los objetivos trazados por el Gobierno Nacional. Según Gabriel Alba (2013): "Un porcentaje muy bajo de niños en el contexto rural estudiado tenía acceso a los videojuegos, y los que tienen lo hacen en sitios de alquiler, los fines de semana cuando van a Bogotá" (p. 83)

Podríamos decir que no hay una coherencia entre lo que pretende alcanzarse y lo que realmente se tiene y se brinda. De nada sirve, por ejemplo, que se entreguen cientos de tabletas en los colegios cuando no se entregan las licencias para que estas puedan ser utilizadas, o que no se tenga la conectividad suficiente, o que se haga pagar a los docentes por el desgaste del material tecnológico. Por otro lado, sabemos que existe la motivación y la capacidad por parte del Gobierno para estar a la vanguardia de las nuevas tecnologías, pero para lograrlo se necesita tiempo y continuidad. Infortunadamente, muchos de los proyectos que se plantean solo se desarrollan durante algunos meses o durante el tiempo del gobierno de turno.

Para que se dé un proceso real de innovación tecnológica, no son necesarios únicamente los equipos y la conexión a internet, también se necesita la educación digital. En un territorio como el latinoamericano, por ejemplo, no basta con anunciar y/o imponer directrices para el desarrollo tecnológico a través del Ministerio de Tecnologías de la Información y las Comunicaciones (MinTic), sino que debe buscarse la manera de propiciar positivamente tales procesos, de lo contrario, se reduce todo a una normatividad sin mayor trascendencia. 
Por su parte, Elisenda Ardevol (2005), en su artículo Cyberculture: Antropological perspectives of the internet, logra abrir una discusión frente a la mirada antropológica que se le puede dar al uso de la red, pues Internet ya ha transgredido los límites como simple herramienta de comunicación y en la actualidad nos damos cuenta que estamos inmersos en el reino de la virtualidad, donde hacemos uso de nuevos términos, de nuevas maneras de comunicación; tenemos, además, nuevas formas de actuar y de pensar que han hecho que cambien las dinámicas de interacción social y con ellas la cultura en sí misma.

Cuando se habla de procesos de innovación tecnológica, generalmente se hace referencia al uso de Internet. Según Ardevol (2005), varios estudios sociales al respecto asumen que:

Un nuevo modelo cultural emergía del uso de Internet que cambiaría los patrones de relación social, la identidad de uno mismo y de la comunidad. Algunos investigadores también pensaron que Internet traería una nueva forma de práctica política y el intercambio económico; por lo tanto, Internet fue vista como una nueva tecnología que afectaría a todas las esferas de nuestra vida. (p. 2)

Si la nueva tecnología afecta todas las esferas de nuestra vida, estamos hablando de una "nueva cultura" emergente que, para algunos enriquece las comunidades, pero para otros, causa el efecto contrario, el cual se evidencia en la pérdida de identidad y pérdida de las culturas autóctonas. Desde ese punto de vista, es preciso preguntarnos: ¿Hasta qué punto estas "nuevas culturas" son buenas para las sociedades? ¿Hasta qué punto se permite un real progreso del país?

En nuestra sociedad colombiana, por ejemplo, a través del MinTic, fundado en el año 2009, se han formulado, adoptado y promovido políticas que buscan la competitividad y avance tecnológico a nivel nacional e internacional; sin embargo, en su intento por impulsar el desarrollo económico del país, ha impuesto procesos que van en contra de la naturaleza de las diferentes comunidades que habitan el territorio colombiano.

Es de suma importancia tener en cuenta que los avances tecnológicos han tardado en llegar a nuestro país, mucha de esa tecnología aún no llega a todas las poblaciones, ni a todas las instituciones educativas por falta de recursos económicos. Todo esto dificulta enormemente el proceso educativo, pues en muchas ocasiones solo se teorizan los avances tecnológicos y no se puede llegar a la aplicación de los mismos.

Dado que es imposible dar la espalda a todas las transformaciones de la sociedad hipermoderna a pesar de las dificultades que estas traen, algunas instituciones han tratado de sobrepasar dichas problemáticas. Es el caso de la Institución Educativa Ricaurte del municipio de Soacha, perteneciente a la comuna 6 , donde el equipo docente se ha dado a la tarea de abrir las puertas no solo a la tecnología, sino también a la preparación de la comunidad educativa para una era globalizada, allí los estudiantes deben llevar la delantera de los cambios a nivel mundial.

En el año 2013, la rectora encargada vio la necesidad de poner su institución a la vanguardia de las tecnologías, así que brindó mejoras en la infraestructura de las aulas de informática, incrementó la cantidad de equipos y, además, comenzó el uso de la plataforma educativa institucional.

A través de dicha plataforma se organizó el material virtual al cual deberían acceder todos los estudiantes diariamente. De la misma manera, los docentes tuvieron la posibilidad de crear sus clases de manera interactiva, innovadora y fácil, pues podían utilizar juegos, videos y música. Así mismo, la evaluación fue mucho más simple para el docente, pues también podían crear sus evaluaciones en línea y la misma plataforma se encargaba de sacar resultados, tabular, hacer estadísticas, entre otras.

Cabe anotar que este tipo de innovaciones ya se han hecho en diferentes instituciones educativas de Bogotá, lo realmente interesante es ver cómo ha sido el proceso que ha desarrollado una institución pública con bajos recursos económicos, cuyos estudiantes pertenecen a estratos uno y dos, en donde sabemos que difícilmente se podría desarrollar un proyecto de innovación tecnológica real o un proyecto bilingüe que vaya de la mano. Al decir "real", se hace referencia a que muchos de los proyectos tecnológicos Ilevados a cabo en el 
municipio de Soacha se conforman con la compra de computadores o tabletas, sin realizar cambios en sus estructuras curriculares o en la forma de dictar las clases, de modo que la mejora no se da, pues al final sigue faltando la mitad del proceso que se necesita para hacer una verdadera innovación.

Evidentemente, los resultados no se hicieron esperar, pues aquellos estudiantes que no habían podido tener acceso a los computadores, ahora podían hacerlo desde su institución. Los estudiantes tuvieron la oportunidad de crear sus propios juegos y ejercicios para estudiar, además de compartir la información con sus compañeros y profesores a través de foros, chats y grupos de estudio. Se vieron más motivados por las actividades del colegio y permanecieron conectados durante varias horas desarrollando las tareas propuestas por los docentes.

Los estudiantes permanecen la mayor parte del tiempo conectados a internet, y aunque muestran un total desagrado por la lectura, es curioso verlos leyendo casi todo el tiempo mensajes instantáneos, o noticias que aparecen en las redes sociales. No es precisamente la lectura la que los cansa y aburre, sino el medio en el cual están escritos los textos. Estamos hablando entonces, de nuevos lectores que poseen características diferentes, y así mismo adquieren habilidades diferentes, por tanto, fue muy acertado haber dado comienzo al proyecto tecnológico en la institución, pues este dio respuesta a las necesidades de nuestros nuevos lectores, de nuestros estudiantes.

Los estudiantes de la Institución Educativa Ricaurte se vieron rápidamente involucrados en el uso de la plataforma, y muchos de ellos mejoraron sus resultados académicos, convirtiéndose además en agentes activos, pues incluso establecieron conexiones entre las asignaturas y construyeron colectivamente con sus compañeros las actividades propuestas, constituyéndose claramente un ejercicio de hipertexto. Recordemos que el hipertexto "es el espacio por excelencia en el que se pueden establecer conexiones y nexos en medio del recorrido por estructuras interconectadas, en las que la red abre espacios de diálogo e interacción" (Riveros, 2012, p. 40), el cual, como herramienta tecnológica, permite al lector cambiar su papel pasivo y convertirse en parte de ese proceso creador. El lector puede fácilmente pasar de ser lector a ser escritor o coautor poniendo al descubierto sus propios escritos y obteniendo rápidamente respuesta a eso que ha escrito.

Pero aquí no acaba todo el proceso, pues la rectora también se ocupó de la formación de los docentes, quienes debían enfrentarse a algo más que una nueva forma de trabajo, a un mundo diferente, con diferente lenguaje $y$, por ende, diferentes formas de comunicación, entre ellas, el chat, las conferencias en línea y las videollamadas. Infortunadamente, la experiencia vivida por los docentes de la institución no fue la misma para todos, pues, aunque muchos de ellos comenzaron satisfactoriamente su capacitación en el uso de plataforma Moodle, muchos otros se resistieron al cambio.

Según esto, podríamos estar hablando perfectamente del fenómeno de migración digital y con ello del concepto de nativos e inmigrantes digitales. Según Prensky (2001), al hablar de nativos digitales e inmigrantes digitales no solo se hace referencia al campo sociológico por el hecho de pertenecer a un territorio específico o el hecho de pasar de un territorio a otro, sino también al campo lingüístico, haciendo referencia al uso de símbolos para comunicarse en un contexto específico, es decir, lengua, nativa si se nace con ella o inmigrante si es aprendida y aún se tiene "acento".

Es claro que Prensky nos hace ampliar el panorama de cómo es vista la tecnología hasta el momento, pues esta traspasa los límites de la ciencia, y tiene que ser estudiada en el ámbito sociológico (cambios en las estructuras sociales), lingüístico (nuevo léxico), cultural (formas diferentes de pensar), psicológico (cambios en el comportamiento) y educativo (formas diferentes de enseñar). De ahí, que podamos identificar a los estudiantes de la institución como nativos digitales, pues ya conocen mucho del vocabulario y en especial del manejo de las nuevas tecnologías, y a los docentes como inmigrantes digitales, quienes deben aprender el nuevo lenguaje y las nuevas formas de interacción.

Teniendo en cuenta lo anterior, podemos afirmar que la tecnología cada día cobra mayor importancia, pues ha incursionado en nuestras vidas vertiginosa y silenciosamente, al punto de poder transformar todo lo que nos rodea. Es por esta razón que apoyo completamente a 
Prensky (2001), en cuanto a que nosotros como docentes debemos hacerle frente a esta gran transformación, comenzar a adaptarnos de la mejor manera al mundo de los estudiantes que nos rodean y así, muy seguramente, mejorar sus procesos de aprendizaje:

Así que, si los educadores Inmigrantes digitales realmente quieren alcanzar a Nativos Digitales -es decir, todos sus estudiantes- van a tener que cambiar. Es hora de que dejen sus quejas, y como dice el lema de Nike de la generación de nativos digitales, “¡Solo hazlo!” (p. 6)

Así, algunos de los docentes de la Institución Educativa Ricaurte debieron comenzar su capacitación desde lo más básico, como el uso de los equipos y el conocimiento de la plataforma (servicios, aplicaciones, cómo crear sus cursos y evaluaciones, entre otros). Poco a poco, como sucede con la lengua extranjera, fueron aprendiendo y desempeñándose cada vez mejor.

Sin embargo, un $20 \%$ de los maestros se resistió al cambio en un comienzo. Algunos argumentaban tal resistencia por considerar el proyecto como otro más de los que suelen llevar a cabo en el municipio, en donde solo se comienza y nunca se termina, o se desarrollan las actividades a medias, sin tener mayor trascendencia.

Otros argumentaron que estaban cansados de recibir extranjeros que les dijeran cómo ser docentes o cómo hacer sus clases sin tener en cuenta el tipo de estudiantes o las condiciones propias, pues mientras en otros países se aceptan máximo 30 estudiantes por aula, en nuestro municipio tenemos que aceptar 50 y a veces más, y no contamos con los mismos recursos y apoyo económico por parte de la Secretaría de Educación del municipio.

Otros, por su parte, expresaron haberse sentido intimidados frente a algo desconocido, el hecho de no saber a qué se iban a enfrentar los ponía en una situación de angustia e intranquilidad. Con el tiempo, dicha problemática se fue desvaneciendo y poco a poco permitió lograr mejores resultados en la puesta en marcha del proyecto tecnológico, pues ahora todos los docentes hablan "un mismo idioma" con sus estudiantes, y su proceso de enseñanza ha cambiado en un $100 \%$.
Por otro lado, algunos maestros aún consideran que el uso de plataformas virtuales pone en riesgo el trabajo literario argumentando que los estudiantes ya no volverán a los libros, por tanto, no llevarán a cabo el tradicional ejercicio de lectura. Sin embargo, esto no sería completamente válido, pues, como ya se dijo, con el manejo de las nuevas tecnologías deben leer todo el tiempo de manera no lineal y colaborativa, es decir, que se hace uso del hipertexto. Así pues, la literatura no se está arruinando, simplemente se están transformando los roles del lector y del autor, simplemente se están Ilevando a cabo nuevas estrategias para la comprensión y expresión de productos literarios novedosos. Así mismo, la tecnología debe ser vista como una herramienta más que permite que el trabajo del docente sea diferente, creativo, innovador y más simple.

Debemos considerar que la literatura no es solo el estudio de obras escritas en papel que fueron hechas en algún momento con cierto cuidado en el uso del vocabulario, forma y estilo, pues esta debe ser vista desde un punto más amplio, ya que no se limita a la comprensión sino también a la expresión del ser. Sin embargo, al hablar de expresión del ser, no podemos considerar que el texto que no contiene formas estilísticas elaboradas deja de ser un texto literario, pues sigue siendo la expresión del sentir humano. Por ejemplo, podemos encontrar varios escritores que han hecho sus obras utilizando vocabulario muy cotidiano, entre ellos encontramos al escritor francés Claude Simon, quien escribe sus obras como un hipertexto: coloca las ideas tal y como van Ilegando a su mente, va conectando ideas a medida que avanza en el relato, va expresando todo lo que siente y como lo siente, es la viva expresión del ser.

Finalmente, los docentes de la Institución Educativa Ricaurte comprendieron que el proyecto iba más allá de la entrega de equipos tecnológicos, que el objetivo era simplificar su trabajo a través de las nuevas tecnologías. De esta manera, los docentes fueron venciendo sus miedos, arriesgándose a la aventura tecnológica.

Visto esto, podemos afirmar que, si realmente nos damos a la tarea de aprender mejor esa lengua digital, esa nueva cultura de nuestros estudiantes, podremos obtener mejores resultados en el proceso de enseñanzaaprendizaje de los educandos favoreciendo la mezcla 
de los contenidos tradicionales con los nuevos campos de acción. Así, coincidimos nuevamente con Prensky (2001) cuando dice: "Los maestros de hoy tienen que aprender a comunicarse en la lengua y el estilo de sus estudiantes" (p. 4).

Es de sumo valor reconocer detalladamente el contexto en el que vivimos y laboramos, para que podamos formular estrategias de enseñanza efectivas, que estén acordes con la realidad de los estudiantes, sin desconocer el avance tecnológico al que nos enfrentamos a diario no solo en el aula, sino a nivel nacional y mundial. También debemos dejar de lado ciertas ideas preconcebidas, como el hecho de ver la tecnología como un enemigo que acabará con la labor docente.

\section{Referencias}

Alba, G. (2013). Los videojuegos en la vida cotidiana de niños, niñas y jóvenes bogotanos. En J. Amador. Infancias, cibercultura y subjetividades (pp. 71-84). Bogotá: Universidad Distrital Francisco José de Caldas.

Ardevol, E. (2005). Cyberculture: Antropological perspectives of the internet. Tomado de: http://www.academia.edu/7527783/ Cyberculture_Anthropological_perspectives_of_the_Internet

Gutiérrez, A. (2003). Alfabetización digital, algo más que ratones y teclas. Barcelona: Gedisa. Tomado de: http://www.terras. edu.ar/biblioteca/2/2GUTIERREZ-MARTIN-Alfonso-CAP-2-Ladimension-digital-de-la-alfabetizacion-multiple.pdf

Lipovetsky, G. (2006). Los tiempos hipermodernos. Barcelona: Anagrama. Tomado de: https://es.scribd.com/doc/81824580/ Lipovetsky-Los-Tiempos-Hipermodernos

Lipovetsky, G. (2010). La Cultura-mundo. Barcelona: Anagrama.

Ministerio de Comunicaciones. Plan Nacional de Tecnologías de la Información y la Comunicación. 2008-2019.
Nos queda una ardua tarea, pues debemos aprender a sacar mayor provecho de las herramientas tecnológicas que llegan día a día, de los nuevos programas y plataformas, y de las formas de trabajo hipertextual y colaborativo, dándonos la oportunidad de conocer más a fondo tanto a nuestros estudiantes como a nosotros mismos, desarrollando nuevas habilidades y destrezas intelectuales, físicas y emocionales. Es imprescindible que el docente abra sus puertas a los cambios culturales, sociales y económicos de las sociedades, ya que de él depende el porvenir de las mismas.

Patterson, N. (2000). Hypertext and changing roles of readers. English Journal, 90 (2), 74-80, noviembre. Tomado de: http:// homepages.gac.edu/ mkoomen/edu241/hypertext.pdf

Pérez, J. (2004). Promoting digital literacy. Barcelona: Universidad Autónoma de Barcelona. Tomado de: http://www.pedz.unimannheim.de/daten/edz-b/gdbk/04/dig_lit_en.pdf

Prensky, M. (2001). Digital natives, digital immigrants. On the Horizon, 9 (5), 1-6, octubre. Tomado de: https://edorigami. wikispaces.com/file/view/PRENSKY+-+DIGITAL+NATIVES+A ND+IMMIGRANTS+1.PDF

Riveros, H. (2012). Hipertexto e interdisciplinariedad: un camino para construir los saberes del futuro. Educación y Ciudad, 22, 33-44, enero-junio. Tomado de: http://www.idep.edu.co/ revistas/index.php/educacion-y-ciudad/article/view/85 


\section{Diálogo del conocimiento}

El texto de esta experiencia va relatando el proceso de incorporación de nuevas tecnologías en una institución, mostrando equilibrio entre lo emprendido y los conceptos y enfoques que va conduciendo el despliegue de la experiencia. De esta manera, a medida que se habla de alfabetización cultural, plataforma digital, nativos digitales, entre otras nociones utilizadas, se va narrando qué ocurre en el colegio con los estudiantes y los maestros. De hecho, la experiencia va mostrando la importancia de relacionar elementos que se vuelven importantes para llevar a cabo los propósitos que emprende.

De este modo se ve necesaria la formación de docentes, la reconfiguración de las clases y las metodologías, cambios en la evaluación, entre otros. Esta manera de dar cuenta de una experiencia es ilustrativa pues nos informa sobre lo que se hace al mismo tiempo que nos indica el sentido y soporte teórico de lo realizado. Además, nos indica que cuando se pone a andar un proceso interesante, para los maestros y la comunidad educativa, poco a poco se van abarcando más aspectos del andamiaje pedagógico.

La escuela hoy tiene que vérselas con los nuevos dispositivos tecnológicos, en tanto que cada vez más hacen parte de la cotidianidad de nuestras vidas. A la escuela le corresponde poner en contacto a los estudiantes con estas herramientas, incorporarlas a sus tareas de socialización de saberes y hacer ajustes metodológicos para que no resulten agregados sin conexión. El artículo aporta elementos en esta dirección y da cuenta, adicionalmente, de algunas vicisitudes que estos procesos conllevan en contexto de una institución educativa. Sin embargo, al reivindicar la incorporación de estas herramientas como medida para dividir la enseñanza y el aprendizaje entre tradicionalista y transformadora, llega demasiado lejos. La incorporación de herramientas tecnológicas puede ayudar, contribuir y facilitar el acceso a la información, pero no define por sí misma la apropiación del saber.

Raúl Barrantes 